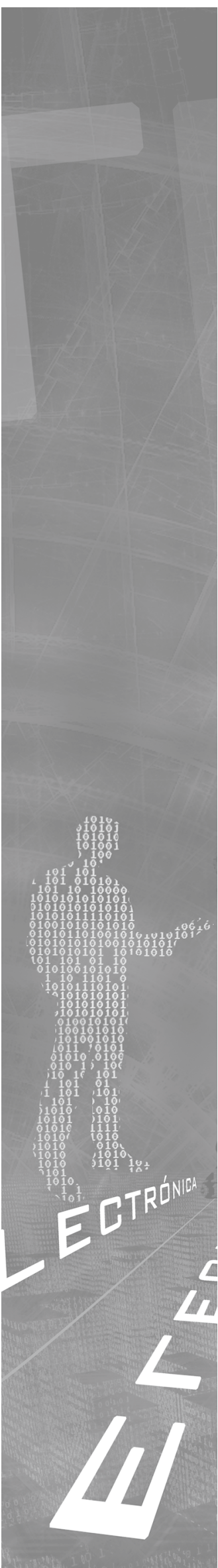

\title{
Proyecto TEACH-Me:
} La experiencia construida en Uniminuto

Grupo de investigación Teach-Me

Recibido el 5 de marzo de 2009. Aprobado el 8 de mayo de 2009

\section{Resumen}

La aplicación de las tecnologías de la información y la comunicación en la enseñanza se ha convertido en un valioso elemento de apoyo didáctico en todos los escenarios de la educación, permitiendo proponer estrategias pedagógicas y didácticas que han apoyado el fortalecimiento de procesos como la comunicación y el trabajo colectivo y asociado (colaborativo]. En este artículo se presenta el proceso desarrollado por la Corporación Universitaria Minuto de Dios -UNIMINUTO, en la implementación del Proyecto TEACH-ME (Technology, Engineering And Calculus Hewlett-Packard Mobile Enviroment), como espacio académico que permite investigar sobre el impacto de la aplicación de las TICs en la enseñanza en la Educación Superior, enfocándose en el proceso desarrollado hasta ahora. Para esto se presentarán los antecedentes del proyecto en UNIMINUTO, el desarrollo que se ha hecho hasta la fecha, el impacto que se ha evidenciado en el aprendizaje del estudiante y la enseñanza, la integración de las distintas tecnologías que han permitido desarrollar el proyecto y por último, los aportes de las Tablet PC al proyecto.

\section{Palabras clave}

Didáctica, Tablet PC, Windows Journal, Inalámbrico, Trabajo Colaborativo, Aula Virtual, Blended Learning.

\section{Abstract}

The application of information technologies and communication in education has become a valuable educational support elements at all stages of education, allowing learning and propose strategies that have supported the strengthening of processes such as communication and collaborative work. This article presents the process developed by the Corporación Universitaria Minuto de Dios-UNIMINUTO in the implementation of the Project TEACH-ME (Technology, Engineering Calculus And Hewlett-Packard Mobile Environment), which allows academic and research on the impact of application of ICT in teaching in higher education, focusing on the process so far. This will provide the background to the project UNIMINUTO, development has been done to date of the project, the impact is reflected in student learning and teaching, integrating technology that has allowed the development of the project and Finally, input from the Tablet PC project.

\section{Key words}

Didactics, Tablet PC, Windows Journal, Wi Fi, Collaborative Work, Virtual Classroom, Blended Learning. 


\section{Introducción}

Las TIC's han incursionado en gran medida en la vida cotidiana de las personas, al punto que nos encontramos en una sociedad donde el conocimiento no es privilegio de pocos, al contrario, la información está al alcance de todos. Los medios de comunicación permiten a las personas, en espacios de tiempo muy cortos, estar al tanto de lo que sucede en lugares remotos. Al observar este fenómeno en el sector educativo, esta nueva concepción de la información y de su uso, debe impactar los procesos de aprendizaje y enseñanza, no solo para ser consecuentes con la realidad sino también, como una estrategia para atraer la atención de los educandos. Como menciona Granados (2005) "Ios sistemas educativos del mundo entero han tenido que replantearse su quehacer: la presencia permanente de docentes y estudiantes en el aula, las clases magistrales, las herramientas didácticas, los textos enciclopédicos que portaban el conocimiento como saberes cerrados, las aulas con infraestructuras físicas espectaculares, el tablero, la tiza, en fin, todo lo que para algunos fue la escuela, la educación y la formación, hoy ha sido revaluado por cientos de instituciones, para ponerse a caminar en paralelo con lo que son las Nuevas Tecnologías de la Información y la Comunicación, TIC's".

La incorporación de las TIC's en la educación no solamente ha sido una preocupación de organismos nacionales, sino también de instituciones internacionales. Por ejemplo Granados (2005) cita un informe de la UNESCO en el que se señala el valor que tienen las TIC's frente al aprender a aprender y al desarrollo de competencias sociales como "el Aprender a Conocer (formación cultural amplia); el Aprender a Hacer, desarrollando la competencia para trabajar en equipo y no sólo limitarse al aprendizaje de un oficio; y el Aprender a Ser, obteniendo mayor autonomía y capacidad para tomar decisiones".

\section{Antecedentes}

A fines de 2006 la multinacional de Informática Hewlett-Packard invitó a UNIMINUTO, a participar en su programa Technology for Teaching (Tecnología para la Enseñanza). Este proyecto consiste en la donación a determinadas entidades educativas, favorecidas por concurso y escogidas por Hewlett-Packard, de una serie de recursos tecnológicos orientados a la enseñanza. Concretamente se trata de equipos portátiles Tablet PC, con capacidades de interconexión inalámbrica, dinero en efectivo, impresoras y otros elementos, cuyo valor total suma US\$ 60,000.

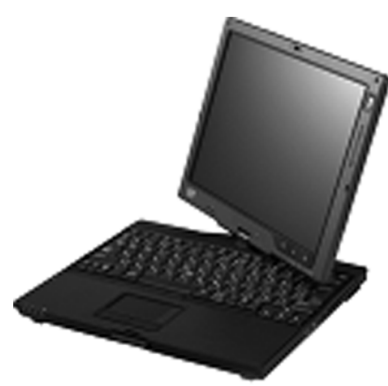

Figura 1.

Si bien concursan instituciones educativas de todo el mundo, solo se puede entrar por invitación. El desarrollo del concurso tuvo una duración de seis meses aproximadamente. El número de instituciones favorecidas no es fijo, debido a que no se tiene en cuenta la cantidad de instituciones premiadas sino la calidad de los proyectos que presentan. En la actualidad hay más de 250 universidades en 36 países que utilizan la tecnología para transformar la enseñanza y el aprendizaje con el apoyo de Hewlett-Packard.

\section{El proyecto TEACH-ME en UNIMINUTO}

El proyecto TEACH-ME' es un espacio de investigación que busca implementar la aplicación de las TIC's en la enseñanza en la Educación Superior, para así proponer estrategias pedagógicas y didácticas que permitan fortalecer procesos como la comunicación y el trabajo colaborativo, bajo la estructura Plan-Programa-Proyecto (Marco Lógico).

Siguiendo esta estructura, UNIMINUTO ha rediseñado los cursos de Gestión Básica de la Información, Fundamentos de Lógica de Programación y Precálculo، cursos pertenecientes a la Facultad de Ingeniería y que son tomados por todos los estudiantes de primer semestre de la misma, buscando como objetivo implementar un ambiente educativo de apoyo al factor presencial, utilizando la herramienta computacional Tablet PC (tecnologías inalámbricas) para contribuir en el proceso de enseñanza - aprendizaje.

Para el desarrollo de este proyecto se han adoptado componentes pedagógicos basados en un modelo constructivista, donde los estudiantes se acercan al conocimiento por medio de sus experiencias en el aula, apoyados en diferentes materiales educativos digitales, los cuales permiten abordar situaciones dinámicas e interactivas. Dentro de este contexto pedagógico los docentes investigadores estudian los

${ }^{1}$ Technology, Engineering And Calculus Hewlett-Packard Mobile Enviroment 
procesos de aprendizaje colaborativo a partir de la incorporación de las TIC's en un entorno donde los estudiantes trabajan juntos resolviendo problemas y desarrollando tareas que permiten aumentar su responsabilidad y autonomía.

La propuesta de UNIMINUTO se basa en desarrollar un aula móvil o itinerante, que desarrolle su actividad en la sede principal durante dos semestres en el año 2008 y en dos regionales los siguientes dos semestres, proyectados para el año 2009. Su objetivo es incorporar el uso de las herramientas computacionales Tablet PC como apoyo a la presencialidad en los cursos rediseñados de Gestión Básica de la Información, Precálculo y Fundamentos de Lógica de Programación, con miras a que al finalizar el proyecto se pueda construir un documento que sirva de guía o mapa de navegación para incorporar las nuevas herramientas en los procesos de enseñanza aprendizaje desde diferentes áreas del conocimiento.

El uso de recursos informáticos, ambientes virtuales de aprendizaje colaborativos, son herramientas que impactan positivamente los procesos de enseñanza aprendizaje de cualquier comunidad, afirmación que busca apoyar este proceso investigativo. Al llevar la tecnología de HP a estos sitios se busca atender las necesidades educativas de estas comunidades, además de cumplir con uno de los principios de UNIMINUTO: "Educación para todos". Por otro lado, intrínsecamente se promoverá el uso de la tecnología para apoyar los procesos de enseñanza aprendizaje, potenciando el crecimiento del mercado a los productos de HP.

\section{Implementación del proyecto en UNIMINUTO}

La investigación que se ha desarrollado es de tipo cualitativo y dadas las características de la población objeto, se ha encaminado como un estudio de caso. Por tal razón, los investigadores se han preocupado siempre por brindar un sustento didáctico, pedagógico, matemático y tecnológico a la intervención en el aula, con miras a ser realmente innovadores y aportar los elementos necesarios a los procesos que se desarrollan en el uso de las TIC's como apoyo a la presencialidad.

El objetivo general del proyecto es aportar a las metodologías de aprendizaje a partir de las Tablet PC de Hewlett Packard, procesos de desarrollo integral, potenciando capacidades cognitivas en los estudiantes de primer semestre de la facultad de ingeniería en Uniminuto.
Para tal fin, durante el segundo semestre de 2007, se hizo una revisión al estado del arte a partir de tres ejes fundamentales:

El tecnológico, específicamente frente al uso de las tecnologías inalámbricas,

El soporte didáctico y pedagógico de la propuesta de aula

- Los componentes matemáticos en relación con la función lineal, el desarrollo de la lógica en el aprendizaje de la programación y los recursos virtuales en la Institución.

La revisión tecnológica se llevó a cabo bajo premisas que permitieran justificar la intervención en el aula usando la herramienta tecnológica Tablet PC de Hewlett Packard, apoyando la presencialidad. Entre estas premisas se revisaron:

- La importancia de las TIC's en la educación superior, Los modelos educativos frente al uso de las TIC's en la formación mixta "blended learning",

- La relación entre la virtualidad como apoyo a la presencialidad y los cambios en el papel de los profesores y los alumnos y la incorporación de las TIC's en los cursos de Precálculo, Programación Básica y Gestión Básica de la Información.

El soporte didáctico y pedagógico de la propuesta se construyó con el fin de justificar la utilización de un modelo teórico validado por la comunidad académica, coherente con los objetivos del proyecto y vinculado con los diversos aspectos que influyen en un proceso educativo. En su elaboración se tuvieron en cuenta las teorías de aprendizaje de Piaget, Vigotsky y Skinner, quienes aportan elementos validos para el diseño de las experiencias de aula y los espacios virtuales de apoyo. Además, se tuvo especial interés en el aprendizaje colaborativo basado en el constructivismo y el uso de las herramientas tecnológicas.

Respecto al componente matemático, lógico y virtual era claro que para hacer propuestas didácticas para la enseñanza de los tres cursos es importante reconocer las representaciones de un objeto de estudio, sus implicaciones en el proceso de aprendizaje, los materiales didácticos necesarios para trabajarlas en el aula de clase y en general, los aspectos que deben ser tenidos en cuenta para potenciar en los estudiantes el estudio y aplicación de la lógica y las matemáticas. Entre los investigadores consultados se encuentran Janvier (1987), Azcárate (2000, 2001), Duval (1996) y Godino, (2005) quienes hacen aportes valiosos en el estudio de las representaciones para el aprendizaje de la lógica y las matemáticas. 
Durante el mismo semestre y con los elementos anteriormente establecidos, se definió la didáctica de la inclusión de los Tablet PC de Hewlett Packard en el aula de clase, para lo cual se tomaron elementos de la Teoría de las Situaciones Didácticas de Brousseau (1986), quien propone cuatro fases fundamentales en las experiencias de aula:

- La acción, donde el alumno actúa sobre el medio, de modo que formula, prevé, y explica la situación; - La formulación, donde se intercambia con una o varias personas información.

- La institucionalización, que permite que el estudiante haga declaraciones que se someterán a juicio de su interlocutor, y

- La validación, en donde finalmente las respuestas encontradas al problema planteado deben ser transformadas para que los conocimientos puedan ser convertidos en saberes.

Para finales del segundo semestre de 2007, se elaboró una propuesta didáctica para el diseño de algunas experiencias de aula, entendidas como la organización dada a todo el desarrollo de una clase, en donde cada paso, cada pregunta, cada momento ha sido planeado y pensado con los siguientes fines: utilización adecuada del tiempo de la clase, acercamiento a los procesos lógicos y matemáticos, planeación de los momentos de actuación del estudiante y del docente, identificación de las producciones del estudiante, establecimiento de conclusiones, fortalecimiento en el uso de las cualidades de la herramienta computacional móvil y registro audiovisual de las experiencias.

Estas experiencias han pretendido orquestarse en el aula a través de la integración entre el blended learning, el aprendizaje colaborativo y la teoría de las situaciones didácticas, de manera que los estudiantes de Precálculo, Programación Básica y Gestión Básica de la Información tengan diversas estrategias y alternativas para resolver situaciones problema utilizando y aprovechando aplicaciones que sólo las herramientas computacionales y virtuales les pueden dar (Tablet PC de Hewlett Packard, espacio virtual en el servidor de Uniminuto), generando un ambiente de asociación en donde los estudiantes aportan ideas y se construye conocimiento como resultado de la interacción de los saberes, el cual, mediado por el docente tiene la función de institucionalizar el saber en el aula a partir de una construcción aceptada por la comunidad.

Se debe aclarar que para los apoyos virtuales requeridos en las experiencias de aula, se aprovechan los espacios de la plataforma virtual de UNIMINUTO,
Moodle, plataforma utilizada en la construcción de cursos basados en Internet y páginas web, haciendo uso de software libre (Open Source).

\section{Estrategias de apoyo al factor presencial}

Hasta el momento se han construido 3 aulas virtuales como apoyo a la presencialidad para los cursos de Precálculo, Programación Básica y Gestión Básica de la Información. Entre los aspectos tecnológicos se enfatizó en elementos propios del blended learning, con el fin de diseñar y generar entornos computacionales que faciliten a los estudiantes la realización de diversas actividades por medio de simulaciones, búsquedas en Internet, uso de multimedia, entre otras, potenciando procesos de gestión de conocimiento tales como: generación, codificación y organización, almacenamiento, acceso y transferencia, todo esto por medio de una enseñanza con tutorías personalizadas, audio, video, blogs, foros y clases presenciales, participando en experiencias de aprendizaje virtual.

En la planificación y construcción de cada aula virtual se han contemplado diferentes etapas descritas a continuación:

\section{Exploración}

Durante esta primera fase se hacen las indagaciones y búsquedas pertinentes en relación con constructos teóricos, teorías del aprendizaje en donde se involucra la didáctica de las TIC's en la educación y el diseño de actividades bajo ambientes virtuales. Se concretan las necesidades del curso, se identifican los recursos, los conocimientos previos y manejo de herramientas de los estudiantes; se determinan las competencias en los contenidos que definirán los objetivos generales del curso.

\section{Identificación de herramientas}

Esta fase permite realizar una búsqueda sobre las diferentes herramientas al nivel de software libre, sus beneficios o inconvenientes, con el fin de establecer los aportes que pueden proporcionar para el desarrollo de diferentes actividades que permitan favorecer el ambiente de aprendizaje.

\section{Diseño}

Esta fase centra el trabajo sobre el análisis de la metodología a implementar, la plataforma tecnológica y en el diseño de las actividades, teniendo en cuenta los elementos didácticos y herramientas virtuales que apoyarán los procesos de aprendizaje, contemplándose también, las competencias y debilidades que 
presentan los estudiantes en sus procesos, lo que permite fundamentar el proceso de investigación.

\section{Implementación}

Esta fase lleva al aula virtual (bajo la plataforma Moodle) las actividades diseñadas, la planeación previa de los contenidos y los recursos (páginas Web, guías de trabajo, videos, evaluaciones, recursos multimedia) a implementar, teniendo en cuenta todos los aspectos con los que cuenta para una oportuna distribución de todo el material de trabajo sobre el aula, además del software que permitirá el desarrollo de algunas tareas.

\section{Aplicación Piloto}

Una vez implementado el ambiente virtual de apoyo a la presencialidad, se realiza la aplicación con un grupo de estudiantes pertenecientes al periodo intersemestral. Con esta experiencia se busca observar el impacto que tengan las Tablet PC en el curso; identificar las fortalezas o debilidades que puedan presentar los estudiantes al desarrollar las actividades planeadas y al utilizar los recursos disponibles.

\section{Retroalimentación}

Una vez analizadas las primeras experiencias, se procede a mejorar o a complementar los recursos, las actividades y demás tareas implementadas en cada uno de los cursos con el fin de mejorar y fortalecer los procesos suscitados previamente.

Para lograr el desarrollo académico dentro del entorno virtual, se hace preponderante que halla total posibilidad de comunicación entre estudiantes y docentes, por esta razón, el uso de elementos como chats y foros es parte indispensable del diseño ya que a partir del exponer, discutir y compartir críticamente los resultados del análisis grupal o individual, el docente puede hacer un proceso de construcción del conocimiento junto con sus estudiantes, logrando que los ambientes de aprendizaje virtuales se conviertan en un medio de comunicación efectivo.

Se pretende que el aula virtual de Teach-Me, se halle caracterizada por un ambiente de fácil manejo, en donde la información sea clara para el estudiante, tanto en la parte del manejo de la plataforma, como de lo que cada docente solicita a manera de trabajos en clase, tareas, actividades de profundización y evaluaciones.

\section{Vl. Impacto en el aprendizaje del estudiante}

El principal impacto originado por la introducción de los Tablet PC de Hewlett Packard en los espacios académicos, se refleja en las bondades que ofrece la tecnología. En los tres cursos implementados que son Gestión Básica de Información, Precálculo y Programación Básica, las conclusiones tomadas de los procesos de observación son similares y se enfocan en:

La posibilidad de desarrollar los diferentes talleres, gráficos y presentaciones en cada uno de los temas tratados, contando con la facilidad de compartir "en línea" con cada uno de los compañeros de la clase, motivando y fomentando el desarrollo del trabajo colaborativo y cooperativo en el estudiante.

El uso de esta tecnología permite mayor atención y concentración por parte del estudiante, eliminando la toma de apuntes en cuadernos, para hacerlo en forma digital, facilitando la presentación o sustentación de trabajos, mejorando la participación en clase y motivando al estudiante a tener una mejor y mayor disposición hacia el aprendizaje.

Las características de conectividad y transmisión de información ofrecidas por los Tablet PC de Hewlett Packard, han resultado ser muy favorables y pertinentes ya que permiten una interacción y comunicación oportuna de las creaciones de los estudiantes. De igual forma, la movilidad de los dispositivos ha permitido que el estudiante asuma un papel mucho más activo dentro y fuera del aula, dado que posibilita el desplazamiento facilitando la constante interacción con los demás integrantes del grupo, permitiendo transformar el aula de clase en un espacio real de aprendizaje y construcción social del conocimiento. Fuera del aula, resulta interesante que los estudiantes puedan explorar en contextos reales, usando la tecnología para modelar su entorno y discutir en el sitio donde él desee, la cafetería, la biblioteca y todo el campus.

\section{Impacto en el encuentro pedagógico}

El desarrollo de un curso teórico-práctico tradicional en UNIMINUTO se apoya en laboratorios de informática previamente acondicionados con el software y los PC de escritorios. Al momento de salir de este formalismo tradicional de los cursos y aplicar las nuevas herramientas tecnológicas que nos facilitan los Tablet PC de Hewlett Packard, de entrada se registra una serie de reacciones de asombro en los estudiantes, cuando en su primer contacto con esta tecnología observan y analizan su movilidad en el salón de clase y aun más cuando experimentan fuera del aula.

La respuesta más común es la de "resulta más cómodo y agradable trabajar fuera del salón de clase". Durante el desarrollo de las diferentes experiencias aca- 
démicas realizadas se ha podido observar las grandes bondades de escritura, de representación de los objetos ya sean matemáticos, algorítmicos o de cualquier otro tipo, y la facilidad de interacción entre el docente y el estudiante que ofrece esta tecnología.

En general, el apoyo de la tecnología inalámbrica, la eliminación del trabajo intramural, el desarrollo de espacios colaborativos de aprendizaje, la interacción y la dinámica de comunicación activa entre docente -estudiantes y estudiante- estudiante, han permitido que el encuentro pedagógico sea más agradable para el estudiante, permitiendo que la temática desarrollada se torne más atractiva para el estudiante, apoyando de esta forma los procesos de asimilación y comprensión.

\section{Integración de tecnología}

Los temas cubiertos en las clases de Gestión Básica de Información, Precálculo y Programación Básica requieren de gran cantidad de componentes prácticos, debido a que su asimilación y comprensión se debe en gran parte a las experiencias desarrolladas. Es por ello que se requieren herramientas que estén acordes con el desarrollo de prácticas que permitan la socialización de las experiencias de los estudiantes fortaleciendo el aprendizaje de los mismos, estos enfoques se están tratando en el proyecto de la siguiente manera:

Como herramientas para comunicación entre los estudiantes y los profesores. Los estudiantes se pueden comunicar entre ellos y con el profesor mediante el uso de aplicativos como Messenger, NetMeeting o DimDim. Apoyados en esta capacidad comunicativa, los estudiantes pueden compartir sus ideas con los demás y generar discusión frente a los temas que se ven en clase.

Como herramienta de exposición de ideas entre estudiantes y profesores. Gracias al uso de aplicaciones como MS PowerPoint y Journal entre otros, los estudiantes pueden exponer de forma abierta sus ideas acerca de los temas vistos en clase.

Como herramienta de entrenamiento. Gracias a la posibilidad de comunicación y exposición mencionada, el profesor puede corregir y revisar la forma como los estudiantes trabajan los temas vistos en clase, haciéndolo de forma abierta (para que todos los estudiantes vean la revisión o corrección) o de forma privada, según se considere.

Para el desarrollo de las prácticas se están utilizando, las siguientes herramientas:

\section{NetMeeting}

Es un cliente de videoconferencia VolP y multipunto incluido en muchas versiones de Microsoft Windows (desde Windows 95 hasta Windows XP ) por medio del cual, se desarrolla interacción sincrónica entre docente-estudiante y estudiante-estudiante. Es usado para compartir aplicaciones, intercambiar información gráfica en una pizarra electrónica, transferir archivos y controlar remotamente otro PC. Estas acciones, aunque sencillas para muchos, son de gran riqueza frente a labores de comunicación grupal en el proceso de construcción del conocimiento.

\section{Dev-C++}

Es un entorno de desarrollo integrado para programar en lenguaje $\mathrm{C} / \mathrm{C}++$, es usado por los alumnos en las practicas de programación básica para desarrollar programas en leguaje C.

\section{Free DFD.}

Es un editor e intérprete de diagramas de flujo. Permite editar, ejecutar y depurar algoritmos representados como diagramas de flujo. Es usado por el docente de programación básica para la enseñanza de algoritmos básicos y complejos de varias dimensiones.

\section{Geogebra.}

Es un software matemático interactivo de geometría dinámica, con el cual se pueden hacer construcciones con puntos, segmentos, líneas, cónicas (a través del ingreso directo con el ratón o mediante instrucciones con el teclado), y todo eso modificable en forma dinámica. Es usado en el curso de Precálculo para definir funciones reales de variable real, calcular y graficar sus derivadas, integrales, etc.

\section{Regla y Compás (C.a.R.)}

Es una aplicación de geometría dinámica la cual simula un compás y una regla. Es usado en el curso de precálculo para la construcción de figuras geométricas en el computador.

\section{Graphmatica}

Es un editor gráfico, interactivo, de ecuaciones algebraicas. Es usado en el curso de Precálculo como una ayuda para graficar. Al mismo tiempo que ha sido diseñado para ser extremadamente simple en su uso.

\section{Máxima}

Es un sistema para la manipulación de expresiones simbólicas y numéricas, incluyendo diferenciación, integración, expansión en series de Taylor, transformadas de Laplace, ecuaciones diferenciales ordinarias, sistemas de ecuaciones lineales, vectores, 
matrices y tensores. Máxima produce resultados con alta precisión usando fracciones exactas y representaciones con aritmética de coma flotante arbitraria. Adicionalmente puede graficar funciones y datos en dos y tres dimensiones.

\section{CmapTools}

Es una herramienta para traspasar a mapas conceptuales conceptos teóricos, de forma que se pueda representar gráficamente una sucesión de ideas. Es usado en el curso de Gestión Básica de la Información para la elaboración orientada de mapas conceptuales, los cuales, una vez han sido concluidos; se pueden transformar automáticamente a formato web para que puedan ser publicados inmediatamente en Internet.

\section{Moodle}

Es un sistema de gestión de cursos de libre distribución que ayuda a los educadores a crear comunidades de aprendizaje en línea. Este tipo de plataformas tecnológicas también se conocen como LMS (Learning Management System). Moodle es usado en el proyecto Teach-Me para la creación de aulas virtuales enfocadas al aprendizaje colaborativo.

\section{Wink}

Es un generador de presentaciones basadas en la grabación mediante video en formato swf de actividades que se pueden realizar en el escritorio del sistema operativo. Es usado para la creación de "video - tutoriales" los cuales son presentados a los estudiantes en el aula virtual de Teach-Me.

\section{Jclic}

Es un entorno multiplataforma para la creación, realización y evaluación de actividades educativas multimedia. Es utilizado para crear actividades interactivas donde se trabajan aspectos procedimentales de los diversos cursos del Proyecto Teach-Me. Esta herramienta hace posible el uso de aplicaciones educativas multimedia "en línea", directamente desde Internet.

\section{Aporte de las Tablet PC}

El equipo del proyecto ha identificado valiosos aportes de las nuevas herramientas tecnológicas, específicamente de los Tablet PC, a los procesos tradicionales de enseñanza aprendizaje. Las características de conectividad y transmisión de información del Tablet PCs-HP han resultado ser muy favorables y pertinentes permitiendo una interacción y comunicación oportuna de las propuestas de los estudiantes.
La movilidad de este tipo de herramientas ha resultado ser sumamente importante a la hora de planear las diferentes actividades, debido a que el docente cuenta con una herramienta ágil para que los estudiantes capturen la información de la práctica y la presenten a sus compañeros, en el momento de hacer la socialización.

El Tablet PC permite al docente registrar y conservar las producciones de los estudiantes de manera instantánea y segura, en formato de sonido y de imagen, teniendo especial cuidado con los procesos argumentativos que desarrollan los estudiantes al resolver diferentes cuestionamientos y que tradicionalmente solo se podía observar de manera escrita, limitando en muchas ocasiones, las producciones de los estudiantes.

La movilidad ha permitido que el estudiante asuma un papel más activo dentro y fuera del aula, posibilitando el desplazamiento y la constante interacción con los demás integrantes del grupo, permitiendo transformar el aula de clase en un espacio real de aprendizaje y construcción social del conocimiento. Fuera del aula, resulta interesante que los estudiantes puedan explorar en contextos reales, usando la tecnología para modelar su entorno y discutir en el sitio donde él desee, la cafetería, la biblioteca, la zona verde, etc.

Para el docente, la movilidad y la captura confiable e instantánea de las producciones de los estudiantes, son los dos elementos más importantes en la búsqueda de mejorar la acción en el aula apoyados en el Tablet PC.

\section{Conclusiones}

- Las experiencias de aula, diseñadas en la investigación y soportadas en la teoría de las situaciones de Brousseau (1986) y el aprendizaje colaborativo, han conformado un ambiente propicio para lograr que los estudiantes elaboren constructos a partir de sus propios conocimientos, haciendo uso de diversos recursos tecnológicos, didácticos y pedagógicos.

- Los resultados de la integración del blended learning como apoyo a la presencialidad, junto con la sustentación pedagógica y didáctica soportada en el uso de las TICs, han permitido identificar y orientar el papel del docente frente a los procesos de aprendizaje, logrando que los estudiantes construyan su conocimiento de forma cooperativa.

- La revisión del estado del arte frente al uso de TICs (especialmente en tecnología inalámbrica) permitió 
potencializar el uso del Tablet PC en contextos fuera del aula promoviendo el aprendizaje colaborativo a través de la comunicación sincrónica.

- La prueba piloto ha permitido evidenciar la posibilidad de replicar en otras asignaturas el trabajo con los Tablet PC dadas sus características, innovación e impacto tanto en los estudiantes como en los docentes.

\section{Referencias}

[1] Azcárate, C. $(2000,2001)$. Funciones. G. P.P. Matemáticas.

[2] Brousseau, G. (1986). Fundamentos y Métodos de la Didáctica de las matemáticas. Traducción hecha por Centeno, Melendo y Murillo, Recherches en Didactique des Mathématiques, Vol. 7 No. 2.
[3] Duval, R. (1996). Semiosis y Pensamiento Humano. Registros semióticos y aprendizajes intelectuales. Edición en Castellano. Colombia: Universidad del Valle. [4] Granados, J. (2005). En Página Institucional del Politécnico Colombiano Jaime Isaza Cadavid. Ponencia: "Las Tecnologías de la Información y la Comunicación en el Politécnico Colombiana Jaime Isaza Cadavid". Bogotá.

[5] Godino, J et al. (2005). Criterios de diseño y evaluación de situaciones didácticas basadas en el uso de medios informáticos para el estudio de las matemáticas. Proyecto de Investigación "Edumat- Maestros", Universidad de Granada. Comunicación presentada en el IX Simposio de la SEIEM, Córdoba (España).

[6] Janvier, C. (1987). Problems of representation in the teaching and learning of mathematics. Université du Québec á Montreal. Canadá: Lawrence Eribaum Associates, Inc., Publishers.

Luis Eduardo Pérez Peregrino. Ingeniero de Sistemas, docente de la Facultad de Ingeniería de la Corporación Universitaria Minuto de Dios (UNIMINUTO).

Adriana Maritza Matallana. Magister en Docencia de las Matemática, docente del Departamento de Ciencias Básicas de la Corporación Universitaria Minuto de Dios (UNIMINUTO).

Frey Rodríguez Pérez, Especialista en Educación Matemática, docente del Departamento de Ciencias Básicas de la Corporación Universitaria Minuto de Dios (UNIMINUTO). 